\title{
New Deployable Membrane Structure Models Inspired by Morphological Changes in Nature
}

\author{
Naoko Kishimoto ${ }^{*}$, M.C. Natori ${ }^{\dagger}$ and Ken Higuchi ${ }^{\ddagger}$ \\ Institute of Space and Astronautical Science / Japan Aerospace Exploration Agency \\ 3-1-1 Yoshinodai, Sagamihara, Kanagawa 229-8501, Japan \\ and \\ Katsuyuki Ukegawa ${ }^{\S}$ \\ Tokyo University, 3-1-1 Yoshinodai, Sagamihara, Kanagawa 229-8510, Japan
}

\begin{abstract}
Some characteristics of morphological changes in nature are discussed and morphological changes in space structure systems are investigated. Essentially space structure systems change their forms and functions, since they must be initially stowed due to spatial constraints of transportation systems, and deployed in their designed orbits. Recently various concepts of membrane structures are proposed for future large space systems, since they can be compactly stowed, and can easily realize space structures with large area. In their developments, it is a major important issue to ensure the reliability of their deployment processes. From the viewpoint of deployment processes, various morphological changes of some plants, insects, and animals are investigated. The efficient characteristics in their morphological changes such as high redundancy, sequential deployment, utilization of gravity forces, and so on are introduced. A new concept of deployable membrane structure models derived especially from the observation of insects' metamorphosis including eclosion of butterflies, dragonflies, cicadas, and so on is proposed. Numerical results of its deployment behavior are also shown.
\end{abstract}

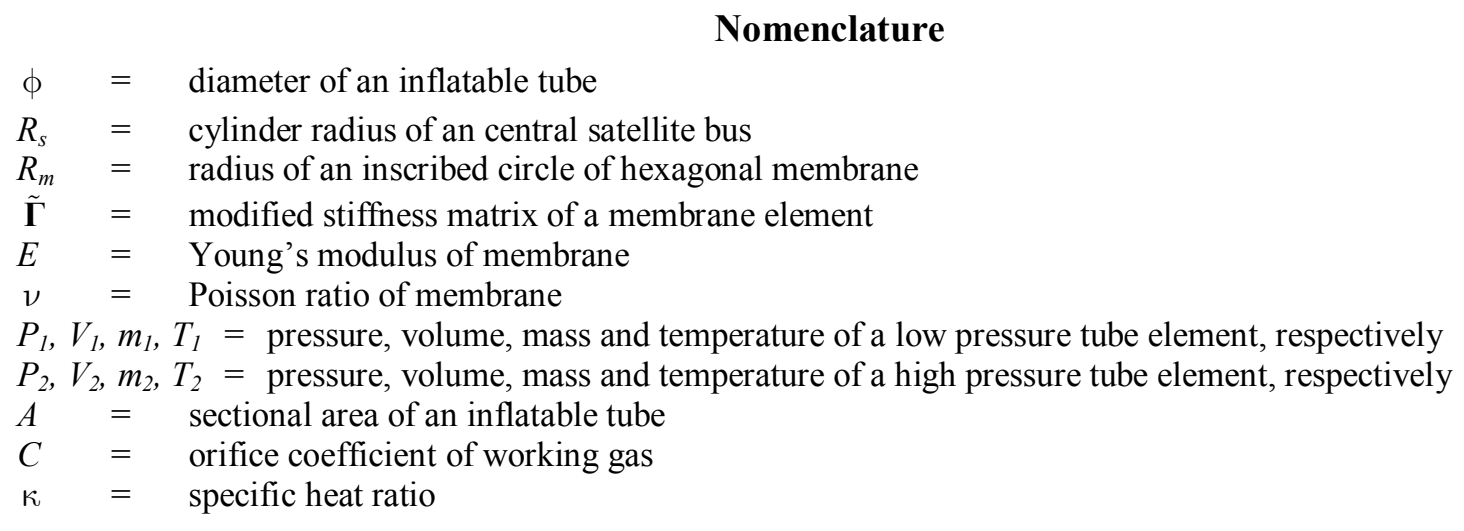

\footnotetext{
* Assistant Researcher, Division of Space Structures and Materials, 3-1-1 Yoshinodai, Sagamihara, Kanagawa 2298510 Japan, and Member AIAA.

${ }^{\dagger}$ Professor and Director, Division of Space Structures and Materials, 3-1-1 Yoshinodai, Sagamihara, Kanagawa 229-8510 Japan, and Associate Fellow AIAA.

* Associate Professor, Division of Space Structures and Materials, 3-1-1 Yoshinodai, Sagamihara, Kanagawa 2298510 Japan, and Member AIAA.

$\S$ Graduate Student, c/o Division of Space Structures and Materials, 3-1-1 Yoshinodai, Sagamihara, Kanagawa 2298510 Japan.
} 


\section{Introduction}

$\mathrm{V}$

arious concepts of membrane structure systems have been proposed for large space structures such as space antennas, solar sails, solar power satellites, and so on ${ }^{1,2}$. Such systems are compactly stowed, and can easily realize space structures with large area. They are effective for space transportation systems having spatial and weight limitation. Membranes are tension members, and usually corresponding compression members are necessary. A representative example of such systems is the square solar sail recently developed especially in the U.S ${ }^{3,4}$. For larger space structure systems over $100 \mathrm{~m}$-scale, usage of centrifugal forces and gravity forces seems to be efficient instead of usage of compression members ${ }^{5}$. These membrane structure systems are naturally deployable structure systems, which can easily cover the necessity of space systems in near future. It is a major important issue to ensure the reliability of their deployment processes.

In order to look for a new possibility of deployable membrane structure systems, we focus on morphological changes in nature. Although current shapes and functions of living things in nature are not always fit for engineering purposes, they show high possibility to adapt to changes of environments ${ }^{6,7}$. One of design principle for biological structural systems is using tensile materials, which reduce total weight ${ }^{8}$. Various morphological changes in plants, insects, and animals are observed. They show some efficient characteristics for deployable structures such as high packaging efficiency, high redundancy, sequential deployment, usage of gravity forces, and so on. Based on these characteristics, we propose a new concept of deployable membrane structure models especially inspired by eclosion processes of insects: they are membrane structures, which embed inflatable tubes. Some examples are introduced for future large planar space membrane structures. Numerical analysis of deployment behavior of a representative model for spinning solar sail is also shown.

\section{Morphological Changes in Nature}

This section treats various morphological changes widely seen in nature. Especially we focus on deployment structures, which involve flowers and leaves in plants, wings of insects and animals. As shown in Table 1, some examples of deployable structures in nature are classified into four groups according to the following two points. One is whether the deployment process is reversible. And the other is whether the deployment process is due to remarkable volume increase.

Table 1 Morphological Changes in Nature

\begin{tabular}{c|c|c}
\hline \hline \multirow{2}{*}{ Deployment only } & \multicolumn{2}{|c}{ With remarkable volume increase } \\
\cline { 2 - 3 } & $\begin{array}{c}\text { Case A: } \\
\text { Bloom of dandelion } \\
\text { Development of new leaves }\end{array}$ & $\begin{array}{c}\text { Case B: } \\
\text { Bloom of morning glory } \\
\text { Eclosion of insects }\end{array}$ \\
\hline & Case D: \\
Both deployment and storage & - & $\begin{array}{c}\text { Bloom of bindweed } \\
\text { Case C: }\end{array}$ \\
& $\begin{array}{c}\text { Open-close movement of sensitive or } \\
\text { insectivorous plants } \\
\text { Flap-stowage of beetle's hind wings } \\
\text { Flap-stowage of bat's wings }\end{array}$ \\
\hline \hline
\end{tabular}

\section{A. Flowers and Leaves (Plants)}

Morphological changes in plants including deployment of flowers and leaves are introduced. There are various types of leaf arrangements, flower crowns, inflorescences. At first, some examples of new leaves are shown in Fig.1. This figure provides that all leaves are initially scale-down and stowed in folded or wound configuration. They gradually grow due to scale-up and deployment processes. Figure 2 demonstrates development of leaves of a legume plant. The total number and arrangement of leaflets are kept through the development, however the size and orientation are gradually changed. These morphological changes are classified into case A in Table 1, because the processes are not reversible and need remarkable volume increase. In addition to this development of leaves, morphological changes in leaves include open-close movement of sensitive or insectivorous plants. The movement is categorized into case $\mathrm{D}$ in Table 1. 

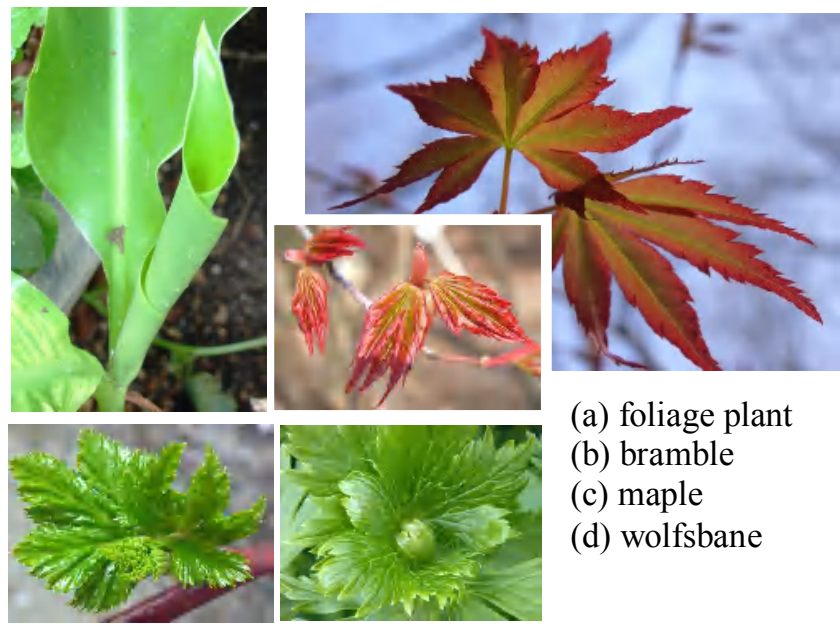
(a) foliage plant
(b) bramble
(c) maple
(d) wolfsbane

Fig. 1 Some examples of new leaves.

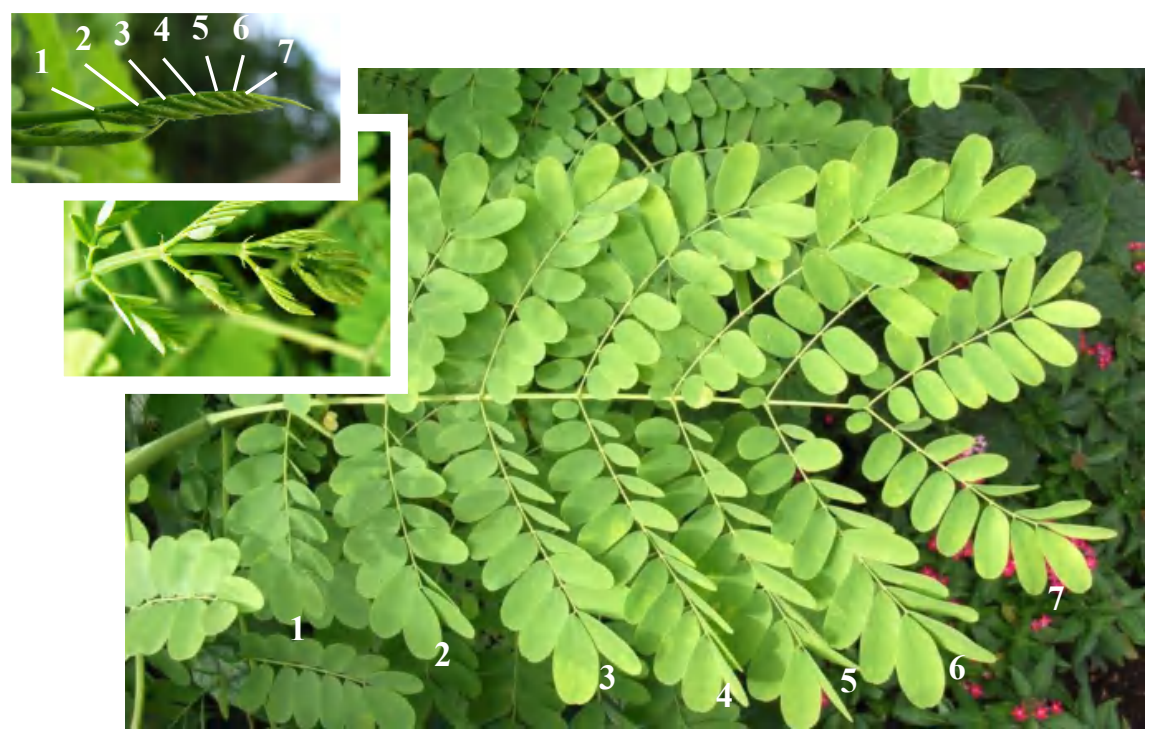

Fig. 2 Development of bipinnately compound leaves of a legume plant.

Next, bloom of flowers is discussed. Figure 3 shows bloom of ladies' tresses. In this case, simultaneous circumferential and vertical bloom forms its unique spiral structure. Bloom of various types of flowers is illustrated in Fig.4. A flower of dandelion(a) or sunflower(b) is composed of tubular and ligulate corolla and deploy their folded petals sequentially. Deployment of dandelion is due to growth of corolla, and it is categorized into case A. Broad petals of poppy are crumpled in its calyx(Fig.4(c)).

Flowers stated above are composed of several corolla or petals. Figure 5 shows bloom of morning glory and
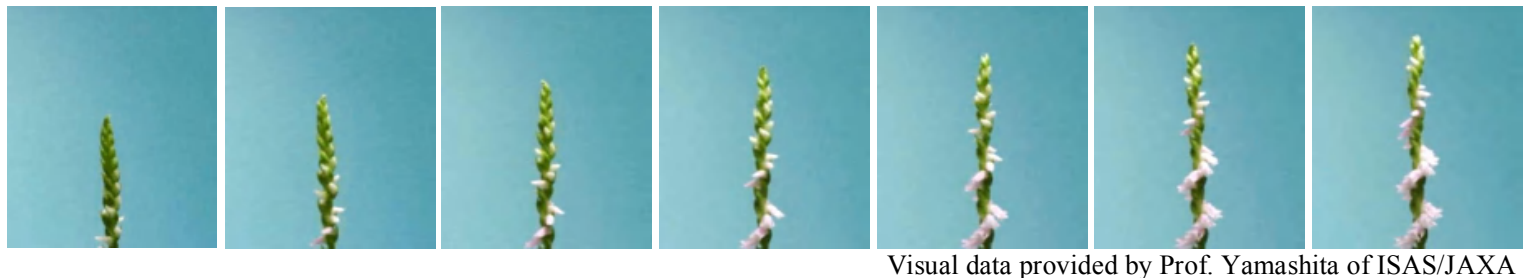

Fig.3 Bloom of Ladies' tresses (Spiranthes sinensis var. amoena).

American Institute of Aeronautics and Astronautics 

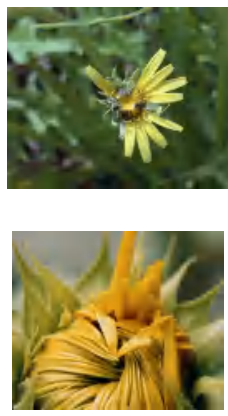

(a) dandelion

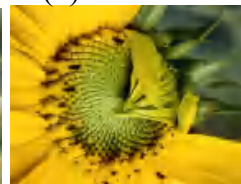

(b) sunflower
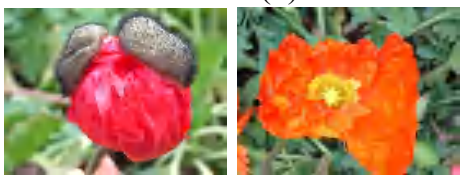

(c) poppy
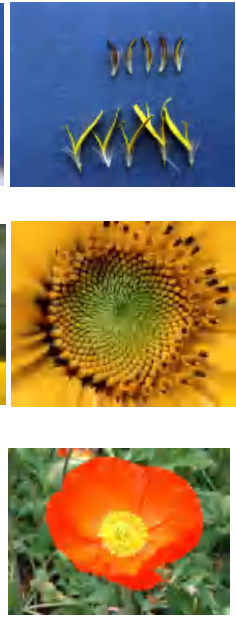

Fig.4 Bloom of various types of flowers.

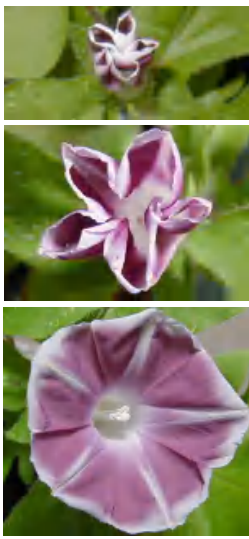

(a) morning glory

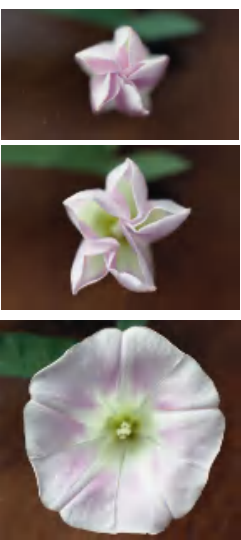

(b) bindweed

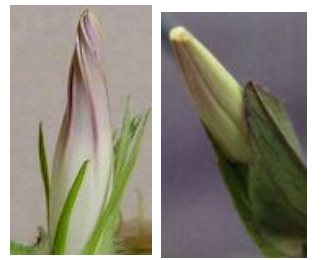

(c) helically-wound buds: morning glory (left) bindweed (right)

Fig. 5 Bloom and helically-wound buds of morning glory and bindweed (infundigular corolla).

bindweed, which flowers are composed of one infundibular corolla. However, morning glory bloom in morning only once, bindweed bloom in the daytime and stowed in the nighttime for a few days. The bloom process of the former is categorized into case B and that of the latter is categorized into case D. Petals of buds of both flowers are wound shown in Fig.5 (c). The petal of morning glory is wound more helically than that of bindweed due to its repeated open-close movements. This feature remains after deployment on the same reason.

\section{B. Insects}

Morphological changes in insects are introduced. Insects need several ecdysis processes, since they have external skeleton. Figure 6 illustrates pupation process of last instar larva of a papilionid butterfly. Morphological changes shown in this section proceed with time from left to right. The shape after the process is very different from that before. Ecdysis and eclosion processes are basically categorized into case B. In addition, morphological changes in insects involve flap-stwrage of beetles' hind wings, which categorized into case D. We give a detailed description of eclosion processes below.

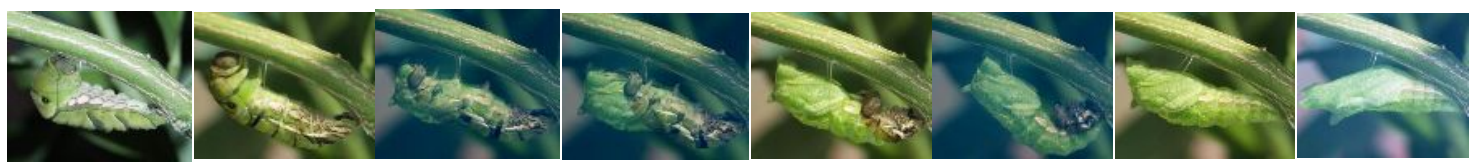

Fig. 6 Pupation process of last instar larva of papilionid (Papilio machaon).

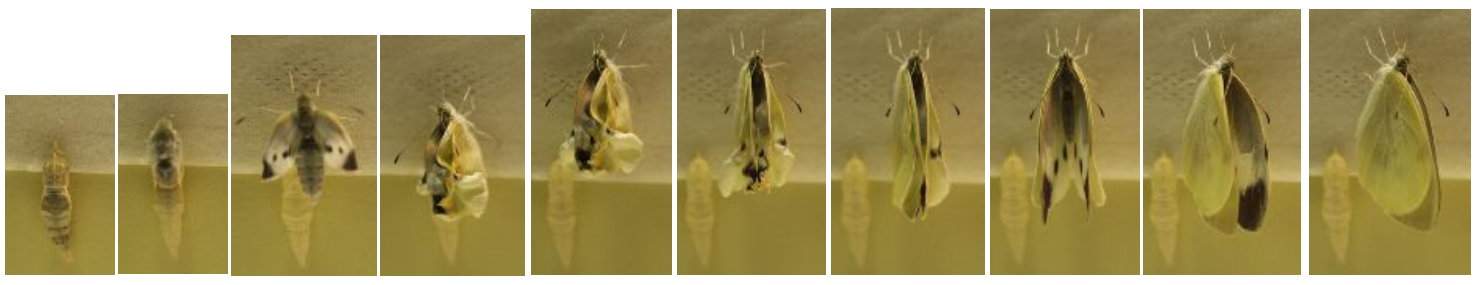

Fig.7 Eclosion process of cabbage butterfly (Pieris rapae).

Eclosion of a cabbage butterfly is shown in Fig.7. Influx of body fluid into branching veins induces deployment of wings. Figure 8 demonstrate eclosion of a nymphalid butterfly, which pupa is hanging head down. In this case, he alters his position during eclosion process. 

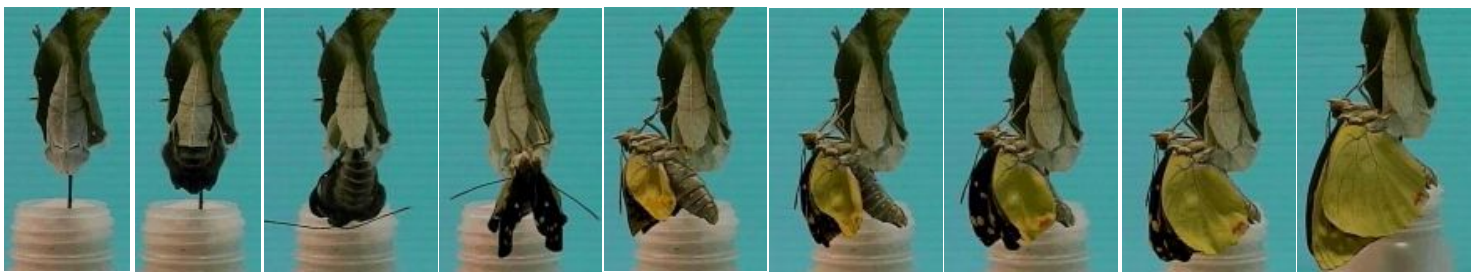

Fig.8 Eclosion process of nymphalid (Sasakia charonda).

Butterflies stated above are complete metamorphosis insects. Figure 9 shows an eclosion process of a dragonfly Anax parthenope Julius, which is an incomplete metamorphosis insect. Four thin membrane wings are stowed in four sheaths on the back of a larva of a dragonfly. A wing consists of thin membrane with many branching veins, and deploys to about forty-fold from its original area. It proves that influx of body fluid into branching veins can expand stowed wings. At last an eclosion process is completed by changing colors of the dragonfly which indicates rigidization of the membranes, veins, and body. Another type of dragonflies' eclosion is shown in Fig.10. In this case, longitudinal axis of wings is perpendicular to the direction of gravity forces.

We point out the following characteristics of the eclosion processes from the viewpoint of structural engineering.
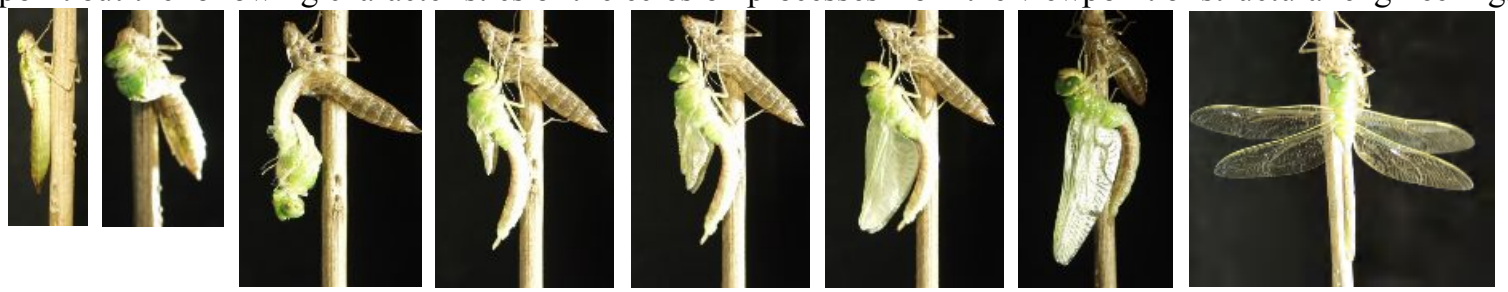

Fig.9 Eclosion process of flying dragon (Anax parthenope Julius).
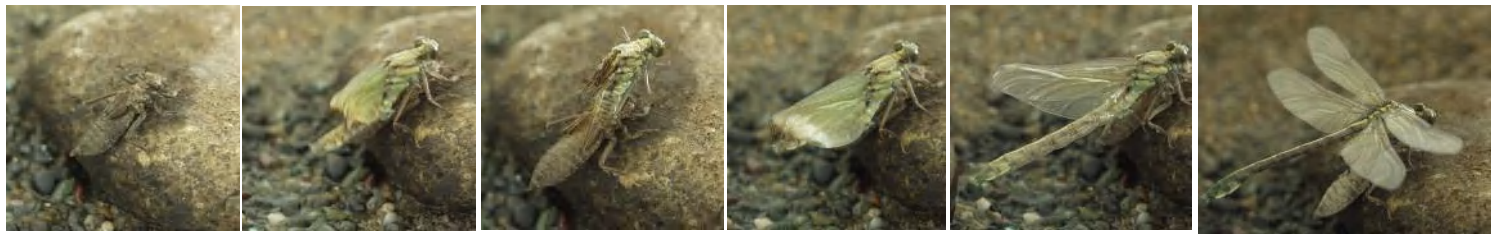

Fig. 10 Eclosion process of Gomphidae (Davidius nanus).

1) Insects' wings are combined structure systems with thin membranes and inflatable veins. And their membranes, veins, and bodies are rigidized after deployment processes.

2) Deployment of a membrane is caused by inflation of branching veins due to influx of body fluid.

3) All body fluid begin to flow from chest of an insect. And it spreads into every edge of wings due to hierarchically branching veins. Diameters of veins become smaller from upper to lower.

4) Dragonflies could use gravity assist in deployment of their membranes, since they change their orientation of body to adjust their directions of expansion of wings to gravity forces.

5) We examine details of a hind wing inside a sheath of a last instar larva of a dragonfly Sympetrum eroticum under microscope. They show that their membranes and veins are folded hierarchically in their sheaths. Hierarchical folding patterns are naturally generated since growth of their membranes is faster than that of their sheaths.

\section{Bats (Animals)}

Lastly we just refer to flaps of bats' wings shown in Fig. 11. They can open and close their membrane wings quickly.

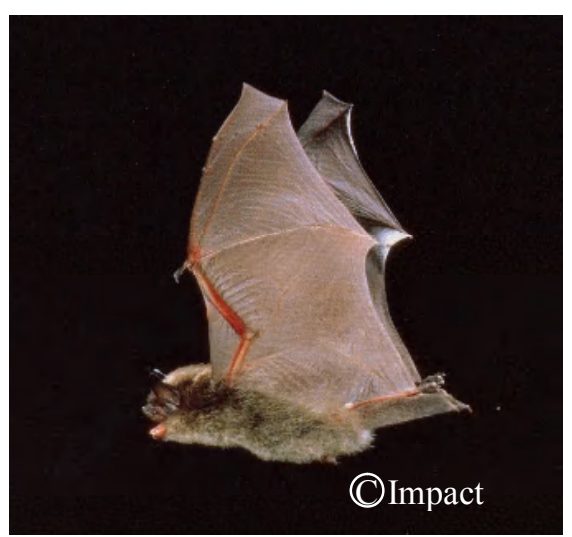

Fig. 11 Flaps of bat's wings. 


\section{Morphological Changes in Space Structure Systems}

\section{A. Consideration of Current Space Structure Systems}

\section{Deployable Structures}

Morphological changes are absolutely essential for space structure systems, since they are launched into space by rocket cargo with spatial and weight limitation. Their representative examples are deployable space structures ${ }^{9,10,11}$. Figure 12 shows exploded view of $\mathrm{M}-\mathrm{V}$ launch vehicle and deployment of a radioastronomy satellite for space VLBI, HALCA, which had a 8-meter mesh reflector supported by tension trusses. As shown in this figure, a rocket vehicle is composed of several parts, such as several stages sequentially separated each other, extensible nozzles, nose fairing, and so on. And it changes its shape after launch. A satellite also changes its shape, since it deploys solar array o mesh reflectors

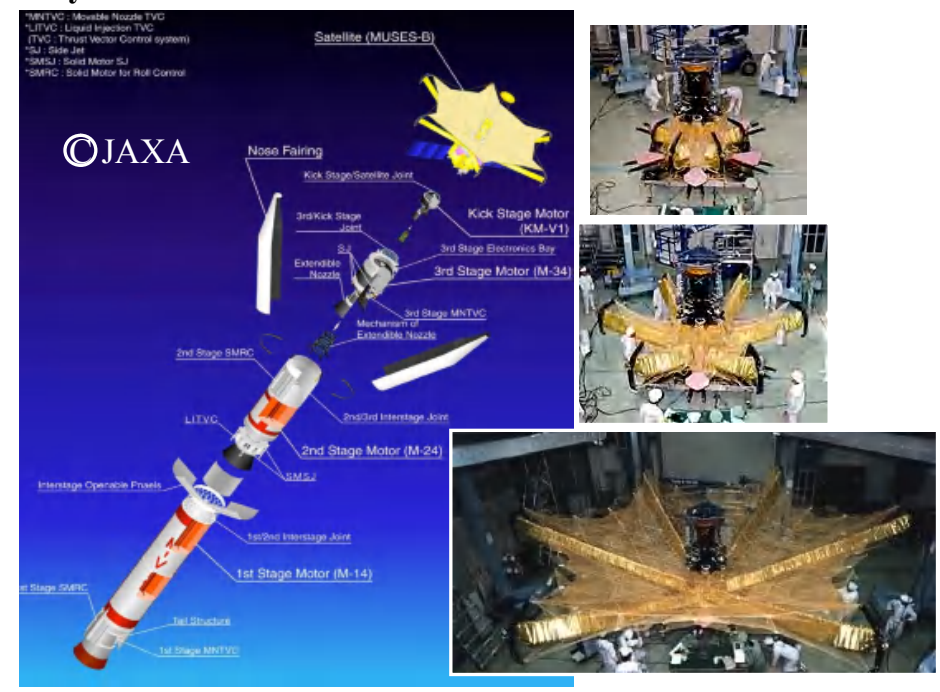

Fig. 12 Exploded view of M-V and deployment of HALCA.

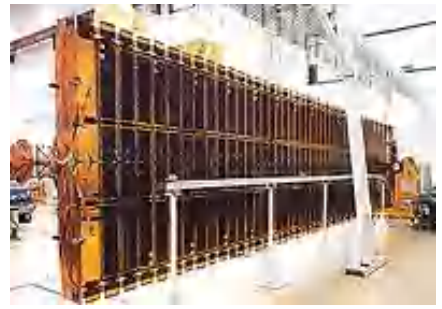

(a) Flexible solar array

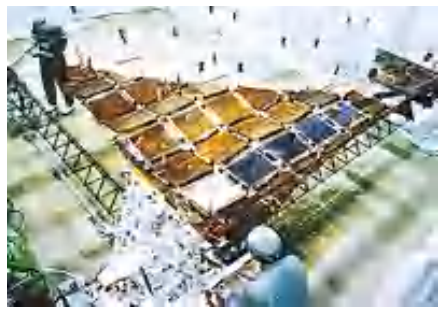

(b) 2-D array

Fig. 13 Deployable solar array.
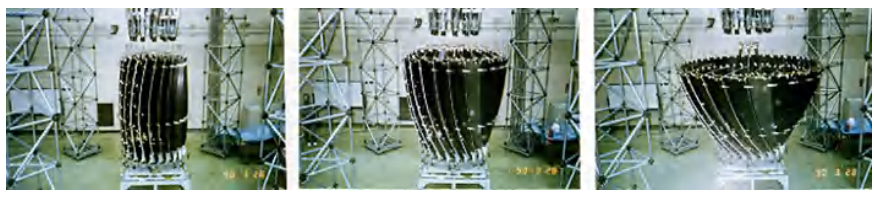

Fig.14 Deployment of a petal type reflector similar to flower bloom.

similar to flower bloom or deployment of leaves already mentioned. Figure 14 shows deployment of a petal type reflector similar to flower bloom. Generally deployable structures for large space systems need elaborate mechanisms shown in Fig. 12. Recently many researches work toward practical use of inflatable structure systems with ultra-light weight and high packaging efficiency $^{1,2}$. A concept of so-called nastic-
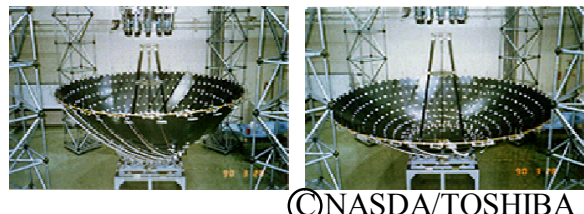

C)NASDA/TOSHIBA

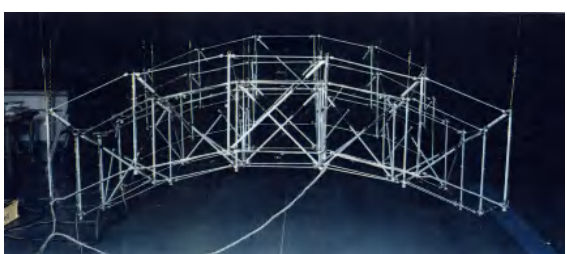

(a) planer VGT

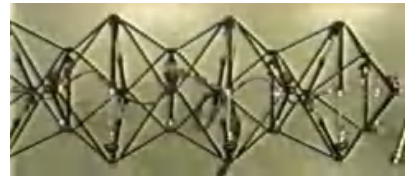

(b) one-dimentional VGT structures is also investigated inspired by the movements of plants due to internal water pressures.

\section{Reconfigurable Structures}

An example of morphological changes in space structures is motion of variable geometry trusses (VGT). Figure 15 shows two examples of VGT, which can change their configuration due to active telescopic members. They are one kind of reconfigurable structures, since their changes are reversible. Reconfigurable structures also include robot manipulators, which are already used for construction of the international space station for examples.

Fig.15 Variable geometry trusses(VGT). 

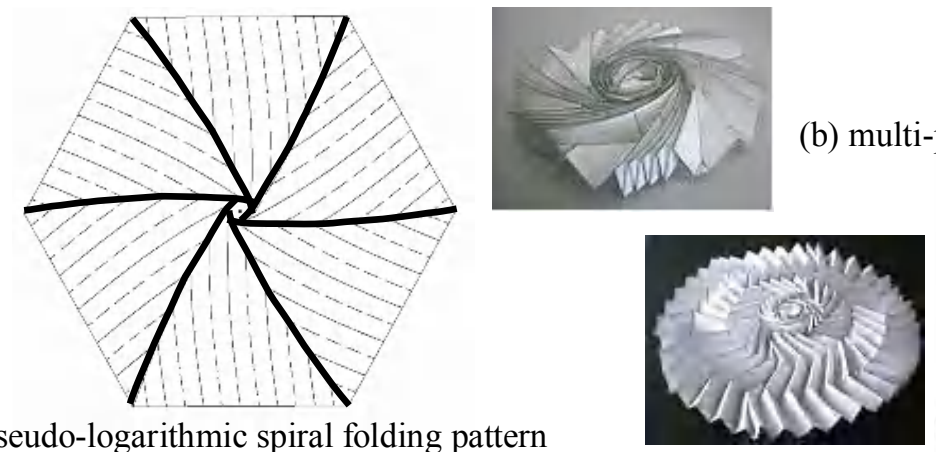

(a) pseudo-logarithmic spiral folding pattern

Fig.16 Example models of proposed membrane structures.

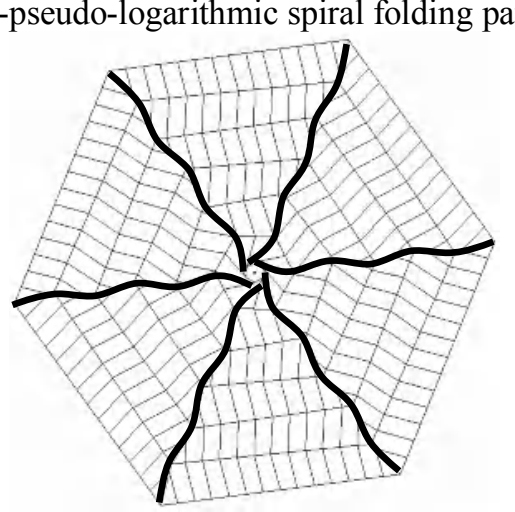

\section{B. New Deployable Structures Inspired by Insects' Eclosion}

1. New Concepts of Deployable Membrane Structures

Considering the characteristics of insects' eclosion among morphological changes in nature stated above, we propose new deployable membrane structure systems, which embed branching inflatable tubes rigidized after their deployment. All inflatable tubes begin from a central satellite bus.

Figure 16 shows two example models of such systems. Heavy lines indicate embedded inflatable tubes. In these cases, inflatable tubes are attached to folding lines of a hexagonal membrane. The both folding patterns are proposed for deployment using centrifugal forces applied to spinning solar sail spacecrafts ${ }^{12}$. We describe characteristics of proposed membrane structure systems in the following.

1) This system needs one tank and device for supplying gas, since all inflatable tubes come from central satellite.

2) We can design geometrical patterns of inflatable tubes without relation to folding patterns. Using branching tubes make deployment process of membranes more smoothly, since number of tubes per an area comes to be constant. Figure 17 shows some examples of geometrical patterns of tubes, which include fractal geometry. These patterns are easily extended to various sizes and shapes of membranes.

3) This system is easily used in combination with other deployable methods such as using deployable masts, centrifugal forces, and so on.

4) We have possibility of controlling deployment processes, since deployment forces due to centrifugal forces are stronger in outer area than inner area, and those due to inflatable tubes are the contrary.

5) Rigidized inflatable tubes provide stiffness and strength to membrane structures. We can provide adequate stiffness distribution of membrane structures by designing branching patterns or distribution of diameters of inflatable tubes (Fig. 17).
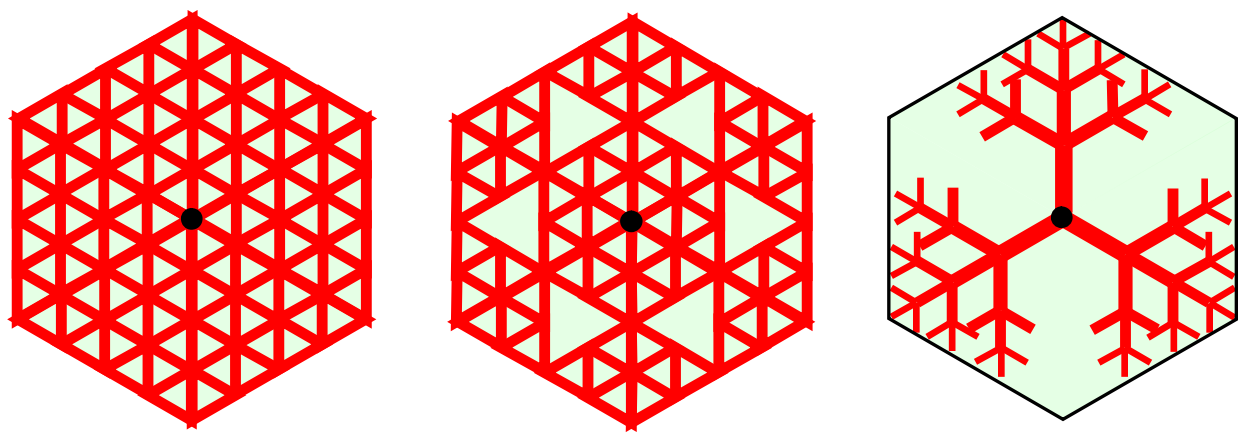

Fig. 17 Three examples of branching patterns of inflatable tubes on hexagonal membrane. 
2. Numerical Analysis of a Hexagonal Membrane Model

In this study, we use one kind of nonlinear elasto-dynamic analysis code developed for numerical analysis of membrane or cable structures ${ }^{13,14}$. This code is characterized by modified stiffness matrix, which means that stiffness becomes 0 or drastically reduced under compression forces. Modified stiffness matrix $\tilde{\boldsymbol{\Gamma}}$ is expressed the following equations.

$$
\begin{gathered}
\tilde{\boldsymbol{\Gamma}}=\frac{E}{1-a_{1} a_{2} v^{2}}\left[\begin{array}{cc}
a_{1} & a_{1} a_{2} v \\
a_{1} a_{2} v & a_{2}
\end{array}\right] \\
\left\{\begin{array}{c}
\text { taut element }:\left(a_{1}, a_{2}\right)=(1,1) \\
\text { wrinkled element }:\left(a_{1}, a_{2}\right)=\left(\eta_{1}, 1\right)
\end{array}\right. \\
\text { slack element }:\left(a_{1}, a_{2}\right)=\left(\eta_{1}, \eta_{2}\right)
\end{gathered}
$$

,where $\eta_{1}$ and $\eta_{2}$ are arbitrary constants greater or equal 0 . In this study, both constants are set to 0 .

Moreover, contact between membrane and satellite body is also considered. Gas flow within a tube is assumed as one dimensional compressive stationary isentropic flow expressed by the following equation.

$$
\dot{m}=C A \sqrt{\frac{2 \kappa}{\kappa-1} \frac{P_{1} m_{1}}{V_{1}}\left(\left(\frac{P_{2}}{P_{1}}\right)^{\frac{2}{\kappa}}-\left(\frac{P_{2}}{P_{1}}\right)^{1+\frac{1}{\kappa}}\right)}
$$

Representative constant data are shown in Table 2 and a model for numerical analysis is shown in Fig. 18.

Deployment behavior of hexagonal membrane models is shown in figure 19. Figure 19 (a) shows the case of deployment without injection and figure 19(b) shows the case with injection. From the comparison between two cases, the case of deployment with injection shows smooth deployment without local deformation. But there appears forced deformation along tube elements. Almost complete deployment accomplishes at 6.0 second. In the case of deployment without injection

Table 2 Constant Data

\begin{tabular}{||c|c||}
\hline \hline membrane material & polyimide \\
\hline Young modulus & $6.963 \mathrm{GPa}$ \\
\hline Poisson ratio & 0.3 \\
\hline membrane thickness & $7.5 \mu \mathrm{m}$ \\
\hline atmospheric pressure & $1.0 \times 10^{-5} \mathrm{~Pa}$ \\
\hline working gas pressure & $1.0 \times 10^{-3} \mathrm{~Pa}$ \\
\hline
\end{tabular}

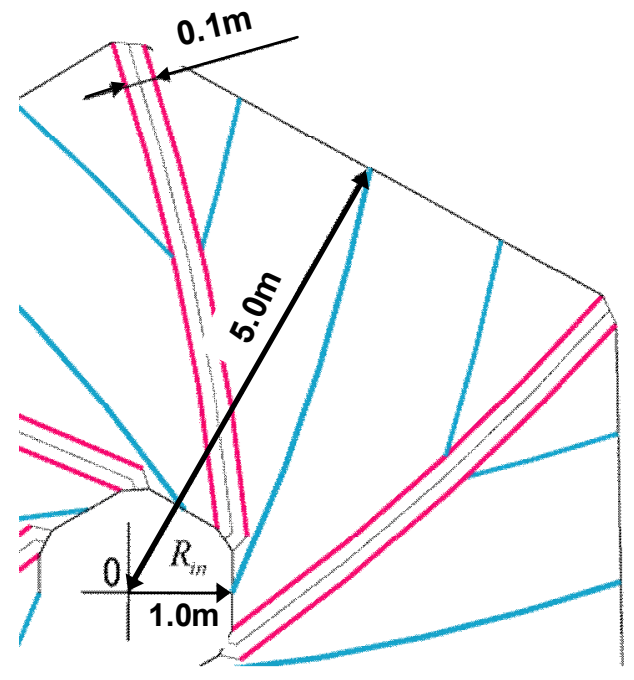

Fig. 18 Analyzed membrane model.
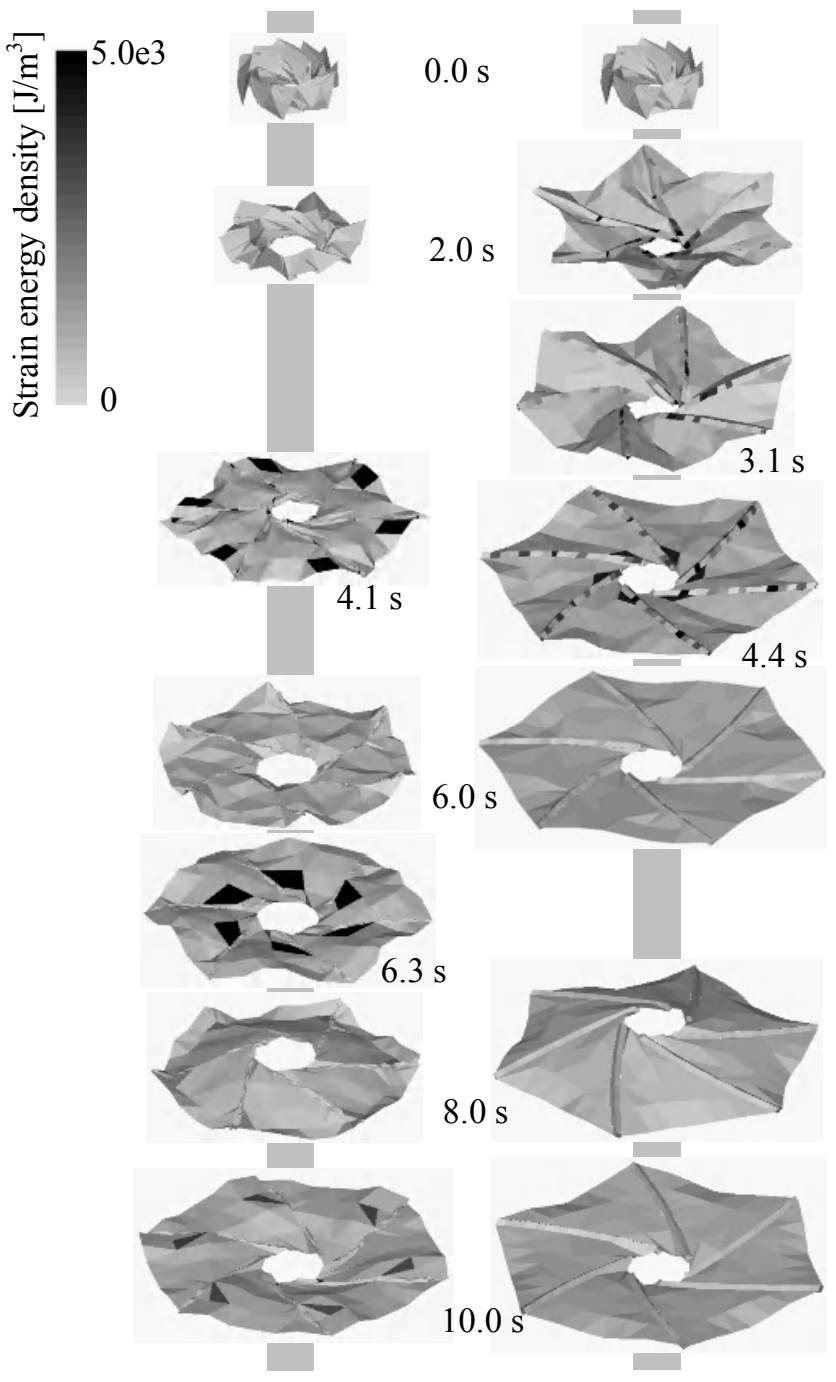

(a) without injection

(b) with injection

Fig. 19 Deployment behavior of hexagonal models. 


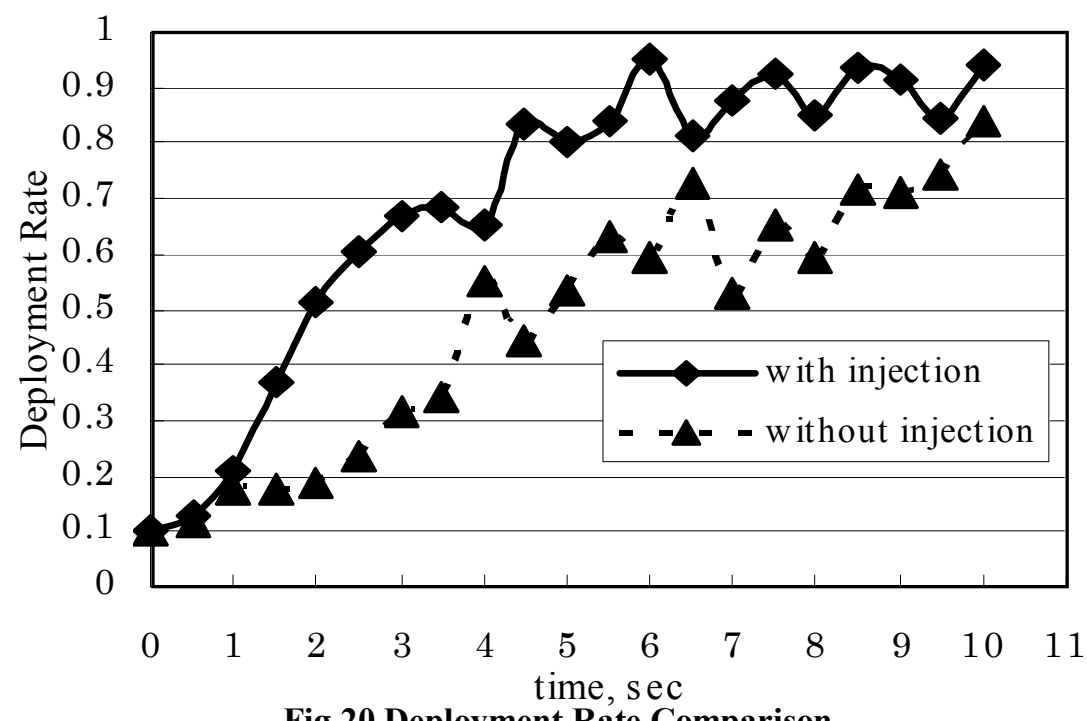

Fig.20 Deployment Rate Comparison.

shown in fig.19(a), local deformation appears and there are many elements with high strain energy density.

Deployment behavior shown in figure 19 is quantitatively evaluated in figure 20, which shows the time history of the rate of deployment area. Overall deployment behaviors with injection and without injection are well presented, and local vibratory behavior is also clarified.

\section{Conclusion}

Deployable structure systems in nature and in space systems are reviewed from the viewpoint of morphological changes. The observation of morphological changes in nature, which include plants, insects, and animals, provides some efficient mechanisms and characteristics to develop deployable structure systems in space systems. Especially we propose a new concept of deployable membrane structure models: they are membrane structures, which embed inflatable tubes. Numerical results of deployment behavior of a representative model indicate its efficiency for smooth deployment process

\section{Acknowledgments}

Professor Yasuyuki Miyazaki, Nihon University, provided invaluable technical assistance for numerical analysis of membrane structures, and numerical results are obtained by Masashi Hashimoto, graduate student of Tokyo University. This work is financially supported in part by Heart Co. Ltd..

\section{References}

${ }^{1}$ Jenkins, C.H.M. (ed.), Gossamer Spacecraft: Membrane and Inflatable Structures Technology for Space Applications, Volume 191 Progress in Astronautics and Aeronautics, AIAA, Virginia, 2001.

${ }^{2}$ Imbriale, W.A. (ed.), Spaceborne Antennas for Planetary Exploration, Deep Space Communications and Navigation Series, Jet Propulsion Laboratory, California Institute of Technology, 2006.

${ }^{3}$ Sleight, D.W., Michii, Y., Lichodziejewski, D., Derbes, B., Mann, T.O., Slade, K.N., and Wang, J.T., "Finite Element Analysis and Test Correlation of a 10-Meter Inflation Deployed Solar Sail," 46th AIAA/ASME/ASCE/AHS/ASC Structures, Structural Dynamics and Materials Conference, AIAA2005-2121, Texas, 2005.

${ }^{4}$ Murphy, D., McEachen, M.E., Macy, B.D., and Gaspar, J.L., "Demonstration of a 20-m Solar Sail System," 46th AIAA/ASME/ASCE/AHS/ASC Structures, Structural Dynamics and Materials Conference, AIAA2005-2126, Texas, 2005.

${ }^{5}$ Natori, M.C., "Response of Structures due to External Forces in Space Environment - A Brief Review- ," Proc. 4th ISAS Space Utilization Symp., ISAS, 1987.

${ }^{6}$ Niklas, K.J., Plant Biomechanics: An Engineering Approach to Plant Form and Function, The University of Chicago Press, Chicago, 1992.

${ }^{7}$ Mattheck, C., Design in Nature: Learning from Trees, Springer, Berlin, 1998. 
${ }^{8}$ Wainwright, S.A., Biggs, W.D., Currey, J.D., and Gosline, J.M., Mechanical Design in Organisms, Princeton University Press, Princeton, 1982.

${ }^{9}$ Thomson, M.W., "The AstroMesh deployable reflector," IUTAM-IASS Symposium on Deployable Structures: Theory and Applications, 2000, pp.435-446.

${ }^{10}$ Freeland, R.E., "Survey of deployable antenna concepts," Large Space Antenna Systems Technology vol.1, NASA CP$2269,1982$.

${ }^{11}$ Guest, S.D., and Pelligrino, S., "A new concept for solid surface deployable antennas," Acta Astronautica, 38, 2, 1996, pp.103-113.

${ }^{12}$ Watanabe, H., Natori, M.C., Okuizumi, N., and Higuchi, K., "Floding of a Circular Membrane Considering the Thickness," ISAS 14th Workshop on Astrodynamics and Flight Mechanics: A Collection of Technical Papers, 2004, pp.19-24.

${ }^{13}$ Miyazaki, Y., and Uchiki, M., "A Numerical Method of Deployment Dynamics of Inflatable Tube," AIAA-2002-1254, 2002.

${ }^{14}$ Miyazaki, Y., "Wrinkle/slack model and finite element dynamics of membrane," International Journal for Numerical Methods in Engineering (in press). 